\title{
Using inpatient data to estimate the prevalence of Wegener's granulomatosis in China
}

\author{
Xiao Liu ${ }^{1.2, *}$, Yazhou Cui ${ }^{1.2, *}$, Yan $\mathrm{Li}^{1.2}$, Chao Wang ${ }^{1.2}$, Heng Zhao ${ }^{1.2}$, Jinxiang Han ${ }^{1.2, * *}$ \\ ${ }^{1}$ Shandong Medicinal Biotechnology Center, Key Laboratory for Biotech Drugs of the Ministry of Health, Key Laboratory for \\ Rare Diseases of Shandong Province, Shandong Academy of Medical Sciences, Ji'nan, Shandong, China; \\ ${ }^{2}$ College of Life Science and Medicine, Ji'nan University, Shandong Academy of Medical Sciences, Ji'nan, Shandong, China.
}

\begin{abstract}
Summary China lacks a registry for most rare diseases, so specific epidemiological data on those diseases are lacking. A strategy involving the DISMOD II model was recently formulated to estimate the epidemiological parameters of rare diseases, and this strategy has been used to study several rare diseases. The current study used this strategy to estimate the prevalence of one such rare disease, Wegener's granulomatosis (WG), in China based on its incidence, mortality, and rate of remission according to the software tool DISMOD II. The incidence of WG was calculated based on inpatient data from 100 hospitals throughout China. The cause-specific mortality from WG was estimated based on data from the National Vital Statistics System of the United States and adjusted for the Chinese population. The rate of disease remission was based on the results of previous study. The current results indicated that the prevalence of WG in China is $1.94 / 100,000$, which is slightly lower than that in Europe and the United States. The mean age at onset of WG in China was calculated to be 38.9 years for males and 39.3 years for females and the duration of disease was 28 years for both male and female patients. These figures are similar to published data from other countries. In conclusion, the DISMOD II model was used to estimate the prevalence of WG in China, providing a basis to evaluate the potential disease burden and orphan drug use by patients with WG. The DISMOD II model could be used to estimate the prevalence of other rare diseases.
\end{abstract}

Keywords: Wegener's granulomatosis, prevalence, rare diseases, DISMOD II, China

\section{Introduction}

Epidemiological data such as disease prevalence is particularly useful in establishing healthcare policies for patients with rare diseases. A conventional sampling approach in an epidemiological study is not suitable for rare diseases because of their extremely low incidence

Released online in J-STAGE as advance publication February $1,2016$.

*These authors contributed equally to this works.

**Address correspondence to:

Dr. Jinxiang Han, Shandong Medicinal Biotechnology Center, Key Laboratory for Biotech Drugs of the Ministry of Health, Key Laboratory for Rare Diseases of Shandong Province, Shandong Academy of Medical Sciences, College of Life Science and Medicine, Ji'nan University, Shandong Academy of Medical Sciences, Ji'nan, Shandong 250062, China.

E-mail: samshjx@sina.com rates. In developed countries such as the United States, patient registries are used to obtain key epidemiological data on rare diseases, but data on only some of those diseases are available. Compared to developed countries, China lacks rare disease registries. Therefore, epidemiological data on most rare diseases are lacking.

DISMOD II is a software tool that is widely used to check the consistency of estimates of incidence, prevalence, duration, and case fatality for different diseases (1). Recently, several studies have formulated a strategy of using DISMOD II to extrapolate the epidemiological parameters of several rare diseases. For example, Yang LP et al. (2) entered incidence, cause-specific mortality, and remission data into DISMOD II to evaluate the prevalence of scleroderma in Spain. Chung et al. (3) used DISMOD II to calculate the epidemiological parameters of multiple sclerosis in South Korea based on its prevalence, incidence, mortality, and duration of disease. Phanthunane et al. (4) 
determined the incidence and duration of schizophrenia based on its mortality, remission, and prevalence in Thailand.

Wegener's granulomatosis (WG) is a typical rare and life-threatening disease with chronic systemic vasculitis that mainly affects the lungs and kidneys (5). In patients with WG, the risk of death increases significantly as the disease progresses, making WG a significant public health problem (6). The estimated prevalence of $\mathrm{WG}$ in Europe ranges from 2.4/100,000 to $15.7 / 100,000$ (7). In the United States, the prevalence of $\mathrm{WG}$ has been estimated to be approximately 2.6/100,000 (8), and in Iran the prevalence of WG is about $0.06 / 100,000$ in children under the age of $15(9)$. However, the prevalence of WG in China has not been known until now.

The current study estimated the epidemiological parameters of $\mathrm{WG}$, i.e. its incidence, mortality, and rate of remission, based on inpatient data from 100 hospitals throughout China. Although such an approach has limitations, it can provide a general view of the epidemiology of WG in China. The results may help to garner more attention and to develop strategies to prevent and reduce the risk of this ignored rare disease.

\section{Data Collection and Methods}

\subsection{Estimation of the incidence of $W G$ in China}

Currently, China has no registry for WG. Data on inpatients with WG in 2011 were used to estimate the incidence of WG in China. Inpatient data were obtained from 100 hospitals in 7 provinces (Shandong, Anhui, Zhejiang, Hebei, He'nan, Jiangxi, and Fujian) in a national pilot project to study rare diseases in China. All data used in this study were accessed in accordance with ethical requirements and did not violate individual rights to privacy. Patients who were diagnosed with WG, i.e. code M 31.3 of the International Classification of Diseases, Tenth Revision (ICD-10), were identified. Clinical data were collected for all patients with $\mathrm{WG}$, including age at the onset of disease, gender, and a confirmed diagnosis of WG. Patients with an unconfirmed diagnosis were excluded. The incidence of WG was estimated based on the number of patients with WG divided by the population served by these hospitals.

\subsection{Estimation of $W G$-specific mortality in China and remission data}

Since data on mortality from WG is currently unavailable in China and other Asian countries, the following strategy was adopted to determine the mortality from WG in China: first, WG is a systemic connective tissue disorder, and information on the total age-specific and sex-specific mortality from systemic connective tissue disorders is available for both China and the United States; second, information on the age-specific and sex-specific mortality from $\mathrm{WG}$ in the United States is available; third, the least squares method was used to predict the mortality from WG in China. US data on WG was used to refine the model. Systemic connective tissue disorders served as an independent variable. Data on Chinese age-specific and sex-specific mortality from WG served as dependent variables.

$X^{k}=\left\{x_{1}^{k}, x_{2}^{k}, \cdots, x_{i}^{k}, \cdots, x_{N}^{k}\right\}$ expresses the independent variable, where $k$ is sex and $i$ is an age variable up to $N$ (in the current study, $N$ was 18).

$Y^{k}=\left\{y_{1}^{k}, y_{2}^{k}, \cdots, y_{i}^{k}, \cdots, y_{N}^{k}\right\}$ expresses the dependent variable, where $k, i$, and $N$ represent the same variables as in the formula for the independent variable. The least squares criterion was used to construct a univariate linear regression model where:

$$
\text { min. } f(a, b)=\sum_{i=1}^{N}\left(y_{i}^{k}-\hat{y}_{i}^{k}\right)^{2}=\sum_{i=1}^{N}\left(y_{i}^{k}-\left(a+b^{*} x_{i}^{k}\right)\right)^{2}
$$

Here, $\hat{y}_{i}^{k}=a+b^{*} x_{i}^{k} \quad$ indicates the fitting results for the independent variable $X^{k}$.

$\mathrm{WG}$ is a rare multisystem autoimmune disease of unknown etiology. According to one study (10), patients with WG may enjoy stable periods from a clinical standpoint, but their return to a healthy state is considered impossible. Based on the criteria in a previous study that defined the rate of remission of scleroderma (11), the current study assumed that the rate of remission of WG was zero.

\subsection{Other data sources}

Population data for 7 provinces by age group and sex was obtained from the Chinese Public Health Data Center. The general age-specific and sex-specific mortality for the entire Chinese population were obtained from the Chinese Center for Disease Control and Prevention.

\subsection{Data analysis using DISMOD II software}

Age-specific and WG-specific mortality, incidence, and rate of remission were entered into DISMOD II. The general age-specific and sex-specific mortality for the entire Chinese population were entered in the tool along with population data. Equations were solved mathematically using the finite differences method, which is a method of iterative approximation. Estimates were calculated assuming a Poisson distribution.

\subsection{Using DISMOD II to estimate the prevalence of hepatolenticular degeneration and hemophilia $A$ in China}

To verify whether the DISMOD II model would be suitable for determining the prevalence of rare diseases 
Table 1. Estimated prevalence of WG in China by age group and gender, based on the cause-specific mortality from WG determined using formulae

\begin{tabular}{|c|c|c|c|c|c|c|c|c|}
\hline \multirow{3}{*}{ Age group } & \multicolumn{4}{|c|}{ Input $(/ 100,000)$} & \multicolumn{4}{|c|}{ Output $(/ 100,000)$} \\
\hline & \multicolumn{2}{|c|}{ Estimated incidence } & \multicolumn{2}{|c|}{ Estimated mortality } & \multicolumn{2}{|c|}{ Prevalence } & \multicolumn{2}{|c|}{ Incidence } \\
\hline & Males & Females & Males & Females & Males & Females & Males & Females \\
\hline $0-4$ & 0.00 & 0.00 & 0.01 & 0.00 & 0.20 & 0.19 & 0.08 & 0.07 \\
\hline $5-9$ & 0.05 & 0.05 & 0.00 & 0.00 & 0.56 & 0.52 & 0.07 & 0.06 \\
\hline $10-14$ & 0.05 & 0.05 & 0.01 & 0.01 & 0.85 & 0.77 & 0.06 & 0.05 \\
\hline $15-19$ & 0.05 & 0.04 & 0.01 & 0.01 & 1.09 & 0.95 & 0.06 & 0.05 \\
\hline $20-24$ & 0.05 & 0.04 & 0.01 & 0.02 & 1.33 & 1.11 & 0.07 & 0.05 \\
\hline $25-29$ & 0.09 & 0.06 & 0.02 & 0.02 & 1.63 & 1.28 & 0.08 & 0.06 \\
\hline $30-34$ & 0.09 & 0.06 & 0.01 & 0.02 & 2.01 & 1.48 & 0.10 & 0.06 \\
\hline $35-39$ & 0.08 & 0.05 & 0.01 & 0.03 & 2.43 & 1.68 & 0.10 & 0.06 \\
\hline $40-44$ & 0.08 & 0.05 & 0.02 & 0.02 & 2.84 & 1.87 & 0.10 & 0.06 \\
\hline $45-49$ & 0.12 & 0.08 & 0.03 & 0.04 & 3.27 & 2.10 & 0.12 & 0.08 \\
\hline $50-54$ & 0.12 & 0.08 & 0.03 & 0.03 & 3.75 & 2.37 & 0.13 & 0.09 \\
\hline $55-59$ & 0.11 & 0.08 & 0.06 & 0.04 & 4.18 & 2.65 & 0.13 & 0.09 \\
\hline $60-64$ & 0.11 & 0.08 & 0.12 & 0.06 & 4.37 & 2.87 & 0.12 & 0.09 \\
\hline $65-69$ & 0.08 & 0.09 & 0.15 & 0.08 & 4.24 & 3.01 & 0.10 & 0.10 \\
\hline $70-74$ & 0.08 & 0.09 & 0.26 & 0.16 & 3.76 & 2.95 & 0.08 & 0.08 \\
\hline $75-79$ & 0.6 & 0.04 & 0.37 & 0.22 & 2.76 & 2.45 & 0.07 & 0.06 \\
\hline $80-84$ & 0.06 & 0.04 & 0.94 & 0.47 & 1.70 & 1.77 & 0.07 & 0.05 \\
\hline $85+$ & 0.00 & 0.00 & 0.00 & 0.00 & 1.50 & 1.63 & 0.02 & 0.02 \\
\hline Total & NA & NA & NA & NA & 2.25 & 1.63 & 0.09 & 0.07 \\
\hline
\end{tabular}

in China, the procedures described earlier were used to estimate the prevalence of hemophilia A and hepatolenticular degeneration in China with DISMOD II. Previous studies have examined the prevalence of these two rare diseases in China (12-13). Therefore, predictions were compared to known prevalence rates for the two diseases.

\section{Results}

The incidence of WG in 2011 was estimated based on inpatient data from 100 hospitals in 7 provinces of China. As shown in Table 1, the incidence of WG increased with age, peaking at 49-74 years for female patients and 25-74 years for male patients. Generally, male patients had an earlier onset and higher incidence of WG than female patients.

Data on mortality from WG is unavailable in China, so the least squares method was used to determine the age- and gender-specific incidence of WG based on data from the United States. Two formulae were derived to predict the mortality from WG in China, and results per 100,000 people in different age groups and with a different gender are shown in Table 1.

(1) For males, the optimal formula was:

$$
\hat{y}_{i}^{\text {male }}=0.0027+0.0446 * x_{i}^{\text {male }}
$$

(2) For females, the optimal formula was:

$$
\hat{y}_{i}^{\text {female }}=0.0033+0.0237 * x_{i}^{\text {female }}
$$

In the two formulae, the R2 statistic was 0.9827 and 0.9734 , respectively, indicating that estimates were highly reliable.

Based on previous studies $(10,11)$, the rate of

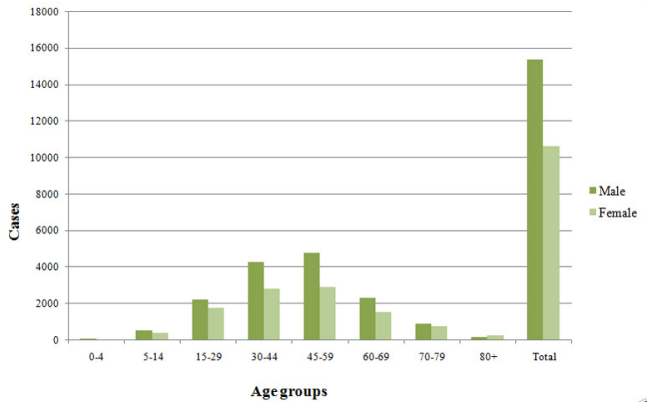

Figure 1. Estimation of the prevalence of WG by age group and gender in China.

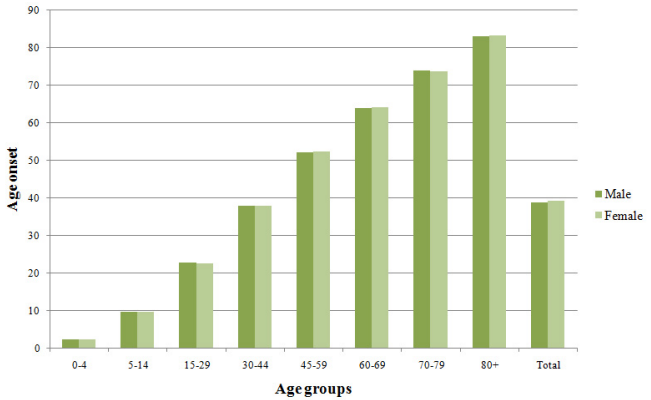

Figure 2. Age of onset of WG by age group and gender in China.

remission of WG can be assumed to be 0 . Based on the estimated incidence, mortality, and rate of remission, the prevalence of WG in China was calculated with DISMOD II to be $2.25 / 100,000$ for males versus $1.63 / 100,000$ for females. There were no significant differences between males and females in terms of the prevalence of WG. The prevalence of WG increased with age and peaked at 60-64 years for males and 65-69 years for females (Table 1). 
After adjusting for the Chinese population, the incidence, mortality, and rate of remission of WG were entered into DISMOD II. China is estimated to have 26,006 patients with WG consisting of 15,384 male and 10,622 female patients. The ratio of males to females in the patient population was 1.45:1 (Figure 1). As seen in Figure 2, the average duration of WG in China was estimated to be 28 years for both male and female patients. In the Chinese population with WG, male patients had an average age of onset of 38.9 years, which is slightly younger than the average age of onset in female patients (39.3 years).

Using the same procedures as were used to analyze WG, the incidence, mortality, and rate of remission of hepatolenticular degeneration and hemophilia A were entered into DISMOD II. The prevalence of hepatolenticular degeneration was estimated to be $2 / 100,000$, which is slightly lower than its actual prevalence $(4.93 / 100,000)$. The prevalence of hemophilia A was estimated to be $2.85 / 100,000$, which is relatively close to its actual prevalence $(2.2 / 100,000)(12-13)$.

\section{Discussion}

Although rare diseases are a topic of growing interest, the epidemiology of rare diseases is still a new area (14). Conventional studies with random sampling are not suitable for studying rare diseases, so epidemiological data on rare diseases is primarily obtained from disease registries or via extrapolation from other data sources. China does not have a specific registry for rare diseases, so important epidemiological data, and especially prevalence, are lacking for most rare diseases (15). A major challenge for China is to develop its medical policies on rare diseases (16-17).

The current study used WG as an example to calculate the prevalence of a rare disease with DISMOD II based on extrapolation. This strategy has been used to study other rare diseases. In the current strategy, three parameters (incidence determined from inpatient data, rate of remission, and estimated mortality) were entered into the DISMOD model. Results indicated that both inputs and outputs had almost the same distribution, so the strategy is highly valid. Moreover, this DISMOD II-based approach was validated with two other rare diseases. The estimated prevalence of these two rare diseases was close to the actual prevalence, suggesting that the current strategy can aptly reflect the actual prevalence of rare diseases. The DISMOD II model could be used to estimate the prevalence and other epidemiological parameters of other rare diseases in China.

Our results indicated that WG has a prevalence of $1.94 / 100,000$ in China, which is close to its prevalence in other countries. This figure is lower than the prevalence of WG in Europe and the United States, where its prevalence ranges from 2.4 to $15.7 / 100,000$, but this figure is higher than the prevalence of WG in Iran $(0.06 / 100,000)$. The incidence of WG in the current study reflects the number of inpatients with WG in proportion to the entire population, so some outpatients who were diagnosed with WG might have been left out. Therefore, the parameters for WG obtained here represent the lower limits, and the estimated prevalence of WG can be viewed as the lower limits of its prevalence in China. Figures for the age at onset and the duration of disease were similar to previously reported figures (10).

Despite the lack of a specific treatment to cure WG, some orphan drugs such as rituximab and methylprednisolone sodium succinate have been found to markedly improve survival. However, most of the orphan drugs to treat WG are still unavailable in China. The estimated incidence and prevalence of WG in China should provide a reference to help authorities develop a specific health policy on WG in the future, such as evaluating the cost-effectiveness of having the healthcare system cover orphan drugs to treat WG. The evidencebased estimates of the prevalence and incidence of WG should also help to encourage industry to research and develop innovative orphan drugs to treat this devastating rare disease.

Although the current results are promising, this study has limitations. First, the effects of some factors, such as socioeconomic status and level of medical care, were not precisely quantified. Second, the exact number of cases of WG throughout the country cannot be readily assessed due to the lack of data from registries. When these data become available, the prevalence of WG and other epidemiological parameters can be estimated more precisely.

In summary, the current study used DISMOD II software to estimate that the prevalence of WG in China. The current results indicated that the prevalence of WG is at least 1.94/100,000 and that China has a total of 26,006 patients with WG. These estimates should serve as an important reference when authorities work to increase the availability of orphan drugs to treat WG and when they develop a medical insurance policy for Chinese patients with WG. The model used here can be used to predict the prevalence of other rare diseases in China.

\section{Acknowledgements}

This work was supported by a grant for Key Projects of the National Program to Support Science \& Technology under the Twelfth Five-year Plan (2013BAI07B02). The authors wish to thank Dr. Chengyu Liu at Shandong University for his kind assistance and suggestions regarding data analysis.

\section{References}

1. Barendregt JJ, Van Oortmarssen GJ, Vos T, Murray CJ. A 
generic model for the assessment of disease epidemiology: The computational basis of DISMOD II. Popul Health Metr. 2003; 1:4.

2. Yang LP, Liang SY, Wang XJ, Li XJ, Wu YL, Ma W. Burden of disease measured by disability-adjusted life years and a disease forecasting time deries model of scrub typhus in Laiwu, China. PLoS Negl Trop Dis. 2015; 9:e3420.

3. Chung SE, Cheong HK, Park JH, Kim HJ. Burden of disease of multiple sclerosis in Korea. Epidemiol Health. 2012; 34:e2012008.

4. Phanthunane P, Vos T, Whiteford H, Bertram M, Udomratn P. Schizophrenia in Thailand: Prevalence and burden of disease. Popul Health Metr. 2010; 8:24.

5. Chen M, Yu F, Zhang Y, Zou WZ, Zhao MH, Wang HY. Characteristics of Chinese patients with Wegener's granulomatosis with anti-myeloperoxidase autoantibodies. Kidney Int. 2005; 68:2225-2229.

6. Luqmani R, Suppiah R, Edwards CJ, Phillip R, Maskell J, Culliford D, Jayne D, Morishita K, Arden N. Mortality in Wegener's granulomatosis: A bimodal pattern. Rheumatology (Oxford). 2011; 50:697-702.

7. Mahr AD, Neogi T, Merkel PA. Epidemiology of Wegener's granulomatosis: Lessons from descriptive studies and analyses of genetic and environmental risk determinants. Clin Exp Rheumatol. 2006; 24(2 Suppl 41):S82-91.

8. Cotch MF, Hoffman GS, Yerg DE, Kaufman GI, Targonski P, Kaslow RA. The epidemiology of Wegener's granulomatosis: Estimates of the five-year period prevalence, annual mortality, and geographic disease distribution from population-based data sources. Arthritis Rheum. 1996; 39:87-92.

9. Tahghighi F, Moradinejad MH, Moradinejad MH, Shiari R, Raeeskarami SR, Salehzadeh F, Javadi V, Ziaee V. Evaluation of 10-year experience of Wegener's granulomatosis in Iranian children. ISRN Rheumatol.
2013; 2013:694928.

10. Watts RA, Al-Taiar A, Scott DG, Macgregor AJ. Prevalence and incidence of Wegener's granulomatosis in the UK general practice research database. Arthritis Rheum. 2009; 61:1412-1416.

11. Villaverde-Hueso A, de la Paz MP, Martin-Arribas MC, Sanchez-Valle E, Ramirez-Gonzalez A, Biairdi P. Prevalence of scleroderma in Spain: An approach for estimating rare disease prevalence using a disease model. Pharmacoepidemiol Drug Saf. 2008; 17:1100-1107.

12. Yanji Qu, Xiaolu Nie, Zhirong Yang, Siyan Zhan. Metaanalysis of the prevalence of hemophilia in mainland China. Chin J Hematol, 2014; 35:65-68. (in Chinese)

13. Wenbin Hu, Yonzhu Han, Renmin Yang, Nan Cheng, Benchun Xue, Daoyin Sun, Xuen Yu, Yongsheng Han, Dongqing Ye. Epidemiological investigation of hepatolenticular degeneration in the Han population of Jinzhai and Lixin counties, Anhui Province: Report of a family pedigree. Anhui Medical Journal. 2012; 33:734737. (in Chinese)

14. de la Paz MP, Villaverde-Hueso A, Alonso V, Janos S, Zurriaqa O, Pollan M, Abaitua-Borda I. Rare diseases epidemiology research. Adv Exp Med Biol. 2010; 686:17-39.

15. Cui Y, Han J. A proposed definition of rare diseases for China: From the perspective of return on investment in new orphan drugs. Orphanet J Rare Dis. 2015; 10:28.

16. Cui Y, Zhao H, Liu Z, Liu C, Luan J, Zhou X, Han J. A systematic review of genetic skeletal disorders reported in Chinese biomedical journals between 1978 and 2012. Orphanet J Rare Dis. 2012; 7:55.

17. Song P, Gao J, Inagaki Y, Kokudo N, Tang W. Intractable and rare diseases research in Asia. Biosci Trends. 2012; 6:48-51.

(Received April 24, 2015; revised December 24, 2015; Accepted December 29, 2015) 